\title{
A unified fielder theory for interception of moving objects either above or below the horizon
}

\author{
THOMAS G. SUGAR, MICHAEL K. MCBEATH, AND ZHENG WANG \\ Arizona State University, Mesa, Arizona
}

\begin{abstract}
A unified fielder theory is presented that explains how humans navigate to intercept targets that approach from either above or below the horizon. Despite vastly different physical forces affecting airborne and ground-based moving targets, a common set of invariant perception-action principles appears to guide pursuers. When intercepting airborne projectiles, fielders keep the target image rising at a constant optical speed in a vertical image plane and moving in a constant optical direction in an image plane that remains perpendicular to gaze direction. We confirm that fielders use the same strategies to intercept grounders. Fielders maintained a cotangent of gaze angle that decreases linearly with time (accounting for $98.7 \%$ of variance in ball speed) and a linear optical trajectory along an image plane that remains perpendicular to gaze direction (accounting for $98.2 \%$ of variance in ball position). The universality of maintaining optical speed and direction for both airborne and ground-based targets supports the theory that these mechanisms are domain independent.
\end{abstract}

Past research into the perceptual strategy used by humans, animals, and robots to navigate to a projectile destination has established that interception can be achieved using only a small number of simple perception-action control heuristics. Here, we examine whether or not the same control heuristics apply when navigating to intercept in the physically vastly different case of targets moving along the ground. If so, this simple strategy can extend to interception from virtually any direction in many domains, both biological and mechanical.

When a fielder runs to catch a ball, a driver navigates to avoid a collision, or a pedestrian intercepts a fellow walker, they each utilize unconscious spatial-orienting principles and other automatic perceptual control mechanisms. At the same time, parallel higher level cognitive processes, such as map building, object discrimination, and conscious analysis of visual streams, are also being performed. Nevertheless, past research on pursuer ability to predict projectile destinations on the basis of knowledge of physics has not been substantiated (Saxberg, 1987; Shaffer \& McBeath, 2002, 2005). For tasks such as interception of moving objects, humans are guided primarily by rapid, unconscious, dynamic, visual-motor control strategies rather than by strategies based on higher level reasoning (Milner \& Goodale, 1995). In this study, we tested whether or not interception of ground balls is

This work was partially supported by National Science Foundation Grants BCS-0318313 and 0403428. The support of Arizona State University and the Arts, Media, and Engineering Laboratory is gratefully acknowledged. We also thank Karen McBeath for editorial assistance, and Igor Dolgov and Crystal Oberle for technical assistance. Correspondence concerning this article should be addressed to T. G. Sugar, Department of Engineering, Arizona State University, Mesa, AZ 85212-0180 (e-mail: thomas.sugar@asu.edu). consistent with usage of only a few simple, perceptually invariant principles.

When catching a moving target, such as a projectile approaching from above the horizon, humans are surprisingly accurate at navigating to the destination, even under varying conditions caused by wind, projectile spin, and other factors. Similarly, when a moving target descends to approach from below the horizon, fielders appear to seamlessly continue their navigational strategy to successfully intercept along the ground. We present evidence to support the parallel use of two general functional heuristics of interceptive action based entirely on control of perceptually invariant principles. This study is the first to utilize high-precision, marker-based, motion-capture technology to unambiguously measure head, body, and target positions during high-speed interception tasks. This type of perceptual modeling is relevant not only for human and animal navigational behavior (Shaffer, Krauchunas, Eddy, $\&$ McBeath, 2004), but also for applied areas such as teleoperation, flight training (Beall \& Loomis, 1997), and mobile robot design (Sugar \& McBeath, 2001a, 2001b; Sugar, McBeath, Suluh, \& Mundhra, 2006).

\section{Perception-Action Control Mechanisms}

Previous research supports the use of navigational mechanisms that guide fly ball interception by maintaining geometric perceptual invariants between pursuer and target projectile. Chapman (1968) and others specified that fielders select a running path that maintains a constant rate of rise for the image of the projectile (Babler \& Dannemiller, 1993; Brancazio, 1985; Chapman, 1968; McLeod \& Dienes, 1993, 1996). More precisely, the control mechanism performs optical acceleration cancellation (OAC) of the tangent of the vertical optical angle (gaze angle). This is geometrically equivalent to the fielder's maintaining a target image that moves upward at a constant velocity 
along a vertical projection plane that moves in depth with the fielder, as is shown in Figure 1A. Since optical gaze angle to the ball increases following a tangent function, the angle itself does not actually increase linearly with time but systematically lessens as the image rises.

Use of this control heuristic can account for interception of target projectiles directed in the sagittal plane toward the fielder. The viability of OAC has also been demonstrated with robotic algorithms (McBeath, Shaffer, \& Sugar, 2002; Mundhra, Sugar, \& McBeath, 2003; Mundhra, Suluh, Sugar, \& McBeath, 2002; Sugar \& McBeath, 2001a, 2001b; Sugar et al., 2006; Suluh, Mundhra, Sugar, \& McBeath, 2002). OAC cannot by itself guide fielders to balls headed off to the side. To do so requires coupling with another mechanism or lateral strategy (Chapman, 1968). For example, researchers suggest that fielders could rotate lateral gaze direction while intercepting fly balls (McLeod, Reed, \& Dienes, 2003).

McBeath and colleagues introduced a simple controltheory model that complements OAC but also explains how fielders determine where to run when catching fly balls headed anywhere on the field (McBeath, Shaffer, \& Kaiser, 1995, 1996; McBeath, Shaffer, Morgan, \& Sugar, 2002; Shaffer \& McBeath, 2002, 2005). The guiding principle is for fielders to run along a path that keeps the ball image monotonically rising along a linear optical trajectory (LOT). Specifically, the ball image is maintained along a directed line relative to the background within successive projection planes that remain perpendicular to fielder gaze direction. Since angular change is defined relative to previous ball position, the ongoing reference frame by which angular change is measured progressively tilts away from the horizon, as described in recent work by us and others (Marken, 2005; McBeath, Shaffer, \& Sugar, 2002). The LOT heuristic is a generic navigational strategy that guarantees interception by utilizing the invariant property of angular constancy between pursuer and target. The LOT control mechanism explains several fielder paradoxes for balls headed to the side, such as why fielders typically limit their speed so they catch the ball on the run
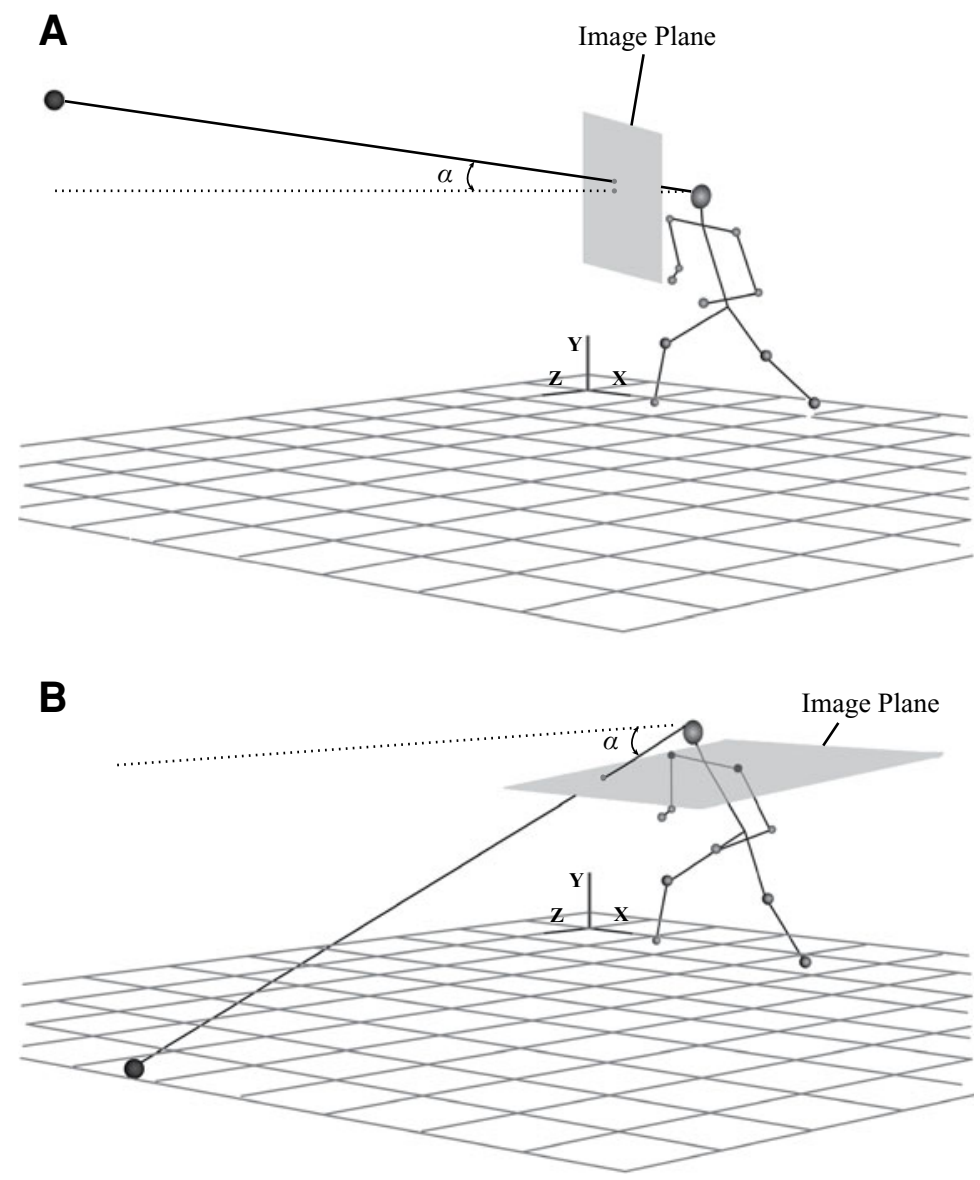

Figure 1. Vertical optical angles and projection planes for fly ball (A) and ground ball (B) cases. When intercepting moving targets, fielders navigate to control $\alpha$, the vertical optical angle between themselves and the target. For fly balls, they keep the image of the ball rising at a constant rate along a vertical projection plane $(\partial \tan \alpha / \partial t=$ constant $)$, whereas for ground balls they keep it approaching at a constant rate along a horizontal projection plane $(\partial \cot \alpha / \partial t=$ constant $)$. 
rather than arrive early, why they pursue uncatchable balls headed well out of bounds, and why balls hit to the side seem easier to catch than balls hit directly toward them. Research confirms that complementary use of OAC and LOT mechanisms is consistent with human and animal interceptive behavior (Shaffer et al., 2004); mathematical, computer, and robot simulations (Aboufadel, 1996; Marken, 1997, 2001, 2005); and cases of fielder failure (Shaffer \& McBeath, 2002).

Humans can determine distance to a stationary object on the ground plane using angle of declination, the angle from the horizon down to the object (Ooi, $\mathrm{Wu}, \& \mathrm{He}$, 2001). Very distant objects appear on the horizon, whereas closer ones have increasingly larger declination angles. We suggest that angle of declination is also systematically used to determine dynamic distance. Other distance cues, such as stereovision, may also become increasingly influential as objects near an observer (Regan, 1997; Regan \& Gray, 2000).

Interception research with ground-based targets also specifies use of lateral optical angle to the target (Cutting \& Wang, 2000; Lenoir, Musch, Janssens, Thiery, \& Uyttenhove, 1999; Pollack, 1995). Pursuers on a lateral collision course typically select a nearly constant bearing path, causing the target to maintain a constant lateral optical speed. Some models define bearing angle as the control variable that is held constant during interception tasks (Fajen \& Warren, 2004; Warren, Kay, Zosh, Duchon, \& Sahuc, 2001). Here, we suggest that control mechanisms similar to those found for fly ball catching also apply for catching of ground balls. We propose that when pursuers try to intercept targets moving below them along the ground, they control relative position using two common, parallel, perceptual mechanisms. The first is OAC, through which the fielder keeps the image of the target approaching at a constant rate along a virtual horizontal projection plane (i.e., the cotangent of declination angle $\alpha$ [ $\cot \alpha]$ continues to decline at a constant rate, as is shown in Figure 1B). The second is LOT, through which the image of the target continues to descend along a constant angle relative to the background on a projection plane that remains perpendicular to fielder gaze.

This proposal is remarkable in that the physics of a ballistic trajectory for a fly ball significantly differ from those of a rolling ground ball. Fly balls travel along airresistance-shortened parabolic trajectories, rising and falling with significant elevation differences due to gravity. In contrast, balls rolling along nearly flat ground are relatively unaffected by gravity, and ground friction is fundamentally different than drag due to air resistance (Watts \& Bahill, 2000). If fielders use the same control strategies to intercept both fly balls and ground balls, then these must be generic, robust, and efficient enough to work in a wide variety of real-world scenarios.

In past studies, navigational perception-action heuristics used in baseball have been tested by recording fielder position and theorizing ball position (McLeod \& Dienes, 1996; Oudejans, Michaels, \& Bakker, 1997). In our early work, we introduced a direct test using a camera mounted on the human or animal fielder (McBeath et al., 1995; Shaffer et al., 2004). In the present study, we introduce the use of multicamera motion capture equipment (Vicon, 2003) to directly measure the high-resolution 3-D positions of target and fielder in real time to test interception strategies.
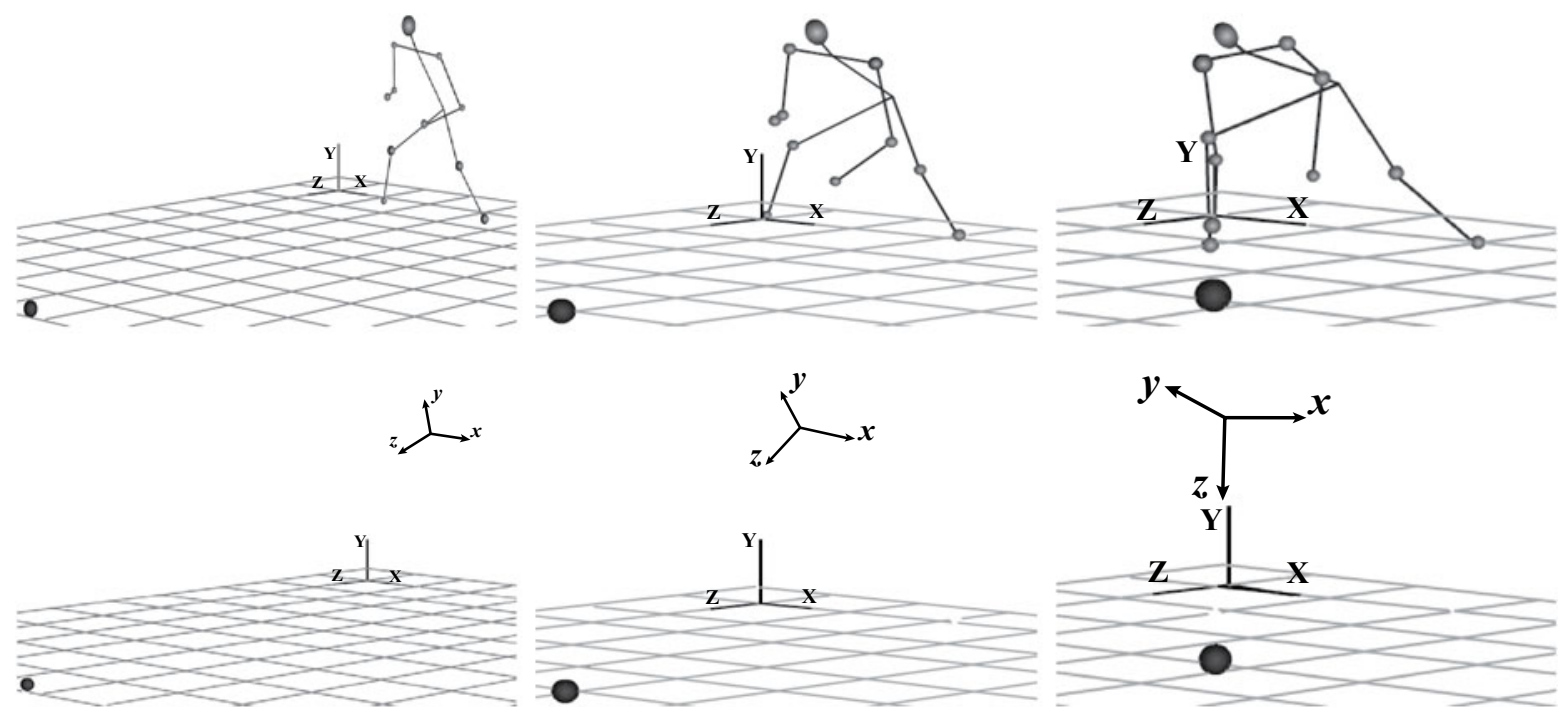

Figure 2. Renditions of three frames from motion capture data collected for a fielder running to catch a ground ball. In the upper panel, a reconstructed stick figure of the fielder is shown standing ready to pursue (left), running forward to intercept (center), and bending over to grasp the target ball (right). Ongoing position data were recorded for the head and the target during the task at exact, synchronous time intervals. In the lower panel, a viewer-based coordinate system is constructed at the fielder's head, with the $z$-axis representing the gaze of the fielder for each of the corresponding top three diagrams. Rotation about the $x$-axis corresponds to declination angle $\alpha$. The (uppercase) $X Y Z$ coordinate system represents the fixed, world-based reference frame of the motion capture system, and the (lowercase) $x y z$ coordinate system represents the moving, viewer-based reference frame. 


\section{METHOD}

\section{Participants}

Three fielders ( 2 amateurs and 1 college-level player) participated in the experiment. All were between 20 and 30 years of age with normal or corrected-to-normal vision. None were aware of the purpose and hypotheses of the experiment.

\section{Procedure}

The participants ran and caught ground balls rolled along a floor. The experiments were conducted in a motion capture laboratory with capture-volume floor dimensions of $10 \times 7 \mathrm{~m}$. The fielders wore suits with retro-reflective markers that were captured by a Vicon eight-camera system with millimeter accuracy at a rate of 120 frames per second. Ongoing 3-D fielder and ball positions were digitized. Balls were rolled at random speeds in random directions within the capture area. Balls were rolled at speeds ranging from 2 to $7 \mathrm{~m} / \mathrm{sec}$ and traveled distances of 3-6 $\mathrm{m}$ before being intercepted. Ball trajectories were angled toward, perpendicular to, and away from the fielder. Difficulty of task varied to include trials in which the fielders had to extend themselves and sometimes move backward. Each trial typically took about 1-2 sec, with the fielder typically running 2 or $3 \mathrm{~m}$ to complete the catch. A total of 30 successful catches by all 3 fielders was recorded.

\section{Analysis Geometry}

Renderings of the raw data from several frames of one trial are shown in the top row of diagrams in Figure 2. The bottom row of diagrams illustrates the viewer-based, body-centered coordinate $(x y z)$ system constructed at the particpiant's head, with the $z$-axis connecting the center of the head with the ball. The $y$-axis remains within the vertical sagittal plane containing the head and the ball, and it is always tilted upward. This is consistent with past findings that gaze rotates forward during interception tasks but the head does not rotate
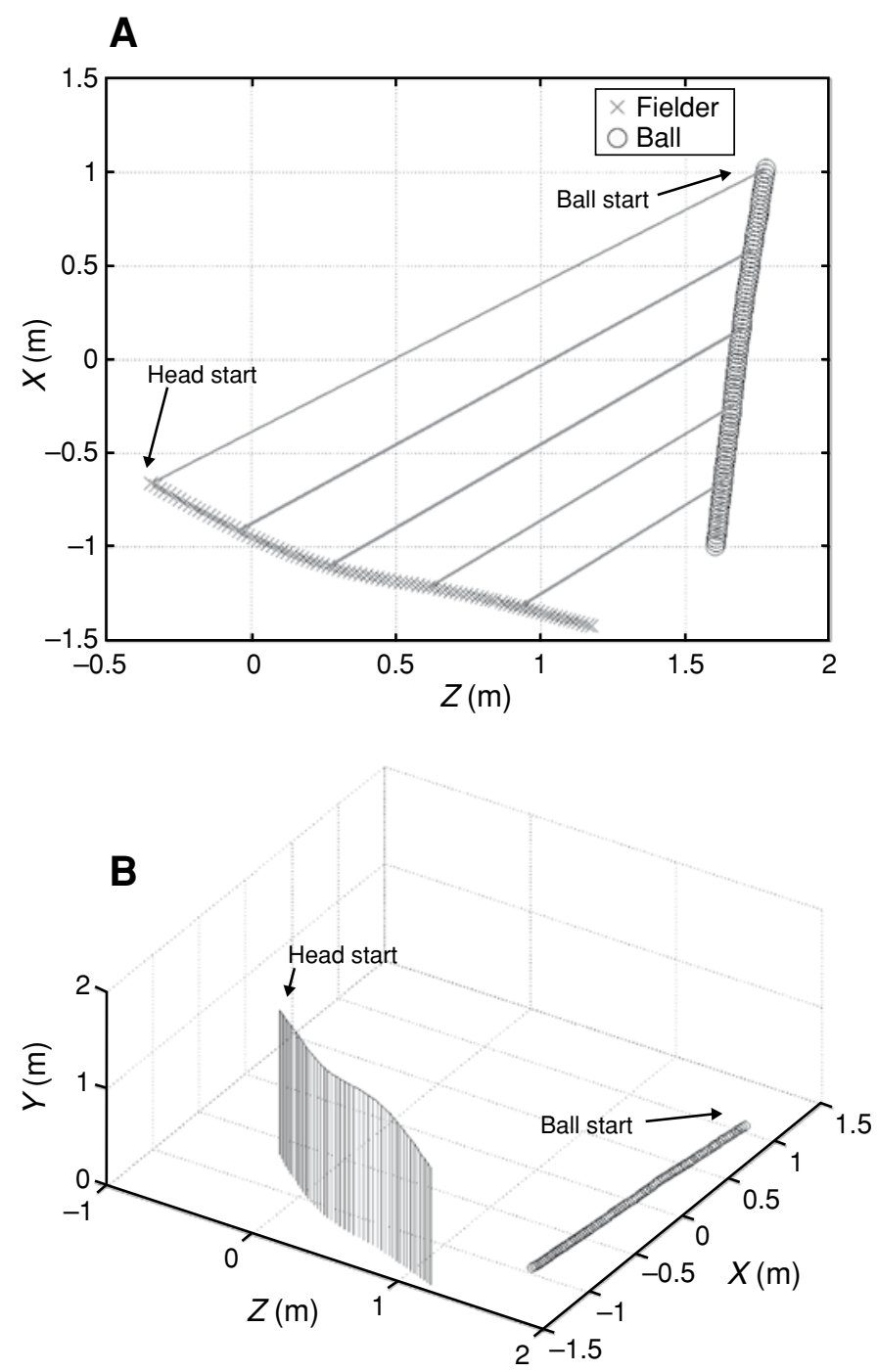

Figure 3. World coordinate data of a fielder intercepting a ball on a typical trial. (A) The fielder's path is indicated by $\times$ s, and the ball's by open circles. The thin, nearly parallel lines represent the gaze direction of the fielder projected onto the world-coordinate $X Z$ ground plane. (B) Three-dimensional plot of the fielder's head and the ball. The vertical lines indicate the fielder's ongoing head location while he approaches the moving ball during the trial shown in panel $A$. 
about the $z$-axis. The raw head-cam data confirm that there was very little sideways twisting of the head. The head-tracking data confirm that the fielders kept their heads and eyes directed at the ball during the interception task, so we used a model of a line directed from the center of the head to the ball. The $x$-axis is constructed to ensure an orthogonal, right-handed coordinate system. The declination angle, $\alpha$, represents rotation about the $x$-axis from eye level, or the angle between the head-to-horizon line and the $z$-axis. The (uppercase) $X Y Z$ coordinate system represents the fixed, world-based reference frame of the motion capture data, and the (lowercase) $x y z$ coordinate system represents the moving, rotating, viewer-based reference frame in which optical linearity is analyzed.

All 30 successful trials were recorded up to the point at which the wrist reached $20 \mathrm{~cm}$ away from the ball. This is because OAC and LOT control mechanisms determine fielder running paths from the starting point to near the destination point, but not for the final grasping task. To model ball-grasping behavior, other timing variables not studied here need to be considered, such as $\tau$ - that is, the time-to-collision estimate from the image-expansion rate of an approaching object (Lee, 1998; Lee \& Reddish, 1981).

\section{RESULTS AND DISCUSSION}

\section{Optical Speed Analysis (OAC Strategy for Interception of Ground Balls)}

Figure 3A shows ground (i.e., $X Z$ plane) trajectories of fielder and ball for a typical trial. Figure $3 \mathrm{~B}$ shows $3-\mathrm{D}$ paths of the fielder's head and the ball. In Figure 3A, the thin lines connecting the two trajectories are gaze directions of fielder projected in the $X Z$ plane at constant time intervals. Alignment angle is defined as gaze direction along the ground plane relative to a fixed coordinate system. Gaze lines remain close to parallel during the trial except at the beginning of the task, despite changes in running speed and bearing angle. Thus, the fielder selects a running path that maintains nearly lateral alignment with the ball. The variable of interest is not magnitude of the lateral angle, but rather its constancy, which is indicated by the fact that the gaze lines remain near parallel. All 30 trials produced nearly constant lateral alignments between fielder and ball, but with a small systematic drift (mean $S D=2.7^{\circ}$ ). In contrast, as is shown in Figure 4, bearing angle (mean $S D=9.4^{\circ}$ ) varied significantly more than alignment angle between fielder and ball $[F(1,29)=$ $11.07, p=.002]$.

Figure 5A shows ongoing changes in height of head, ball, and wrists, with notable changes in head elevation. Figure 5B illustrates the precision of the linear function of optical gaze position despite bouncing of head and ball. Even though head height changes dramatically, declination angle $\alpha$ varies smoothly, as predicted by OAC control strategy. It is clear that $\cot \alpha$ varies linearly with time, and not angle $\alpha$ itself, as is illustrated in Figure 5C. The results were duplicated in a large real-world outdoor setting with bouncing balls, in which fielders maintained a smoothly decreasing declination angle (McBeath, Sugar, Thompson, \& Mundhra, 2003). Clearly, fielder head movement occurs in a manner that maintains optical speed constancy.

For all 30 trials, the OAC control model of maintaining a constant rate of change of $\cot \alpha(\partial \cot \alpha / \partial \mathrm{t}=$ constant $)$ accounted for a median of $98.7 \%$ variance in optical ball
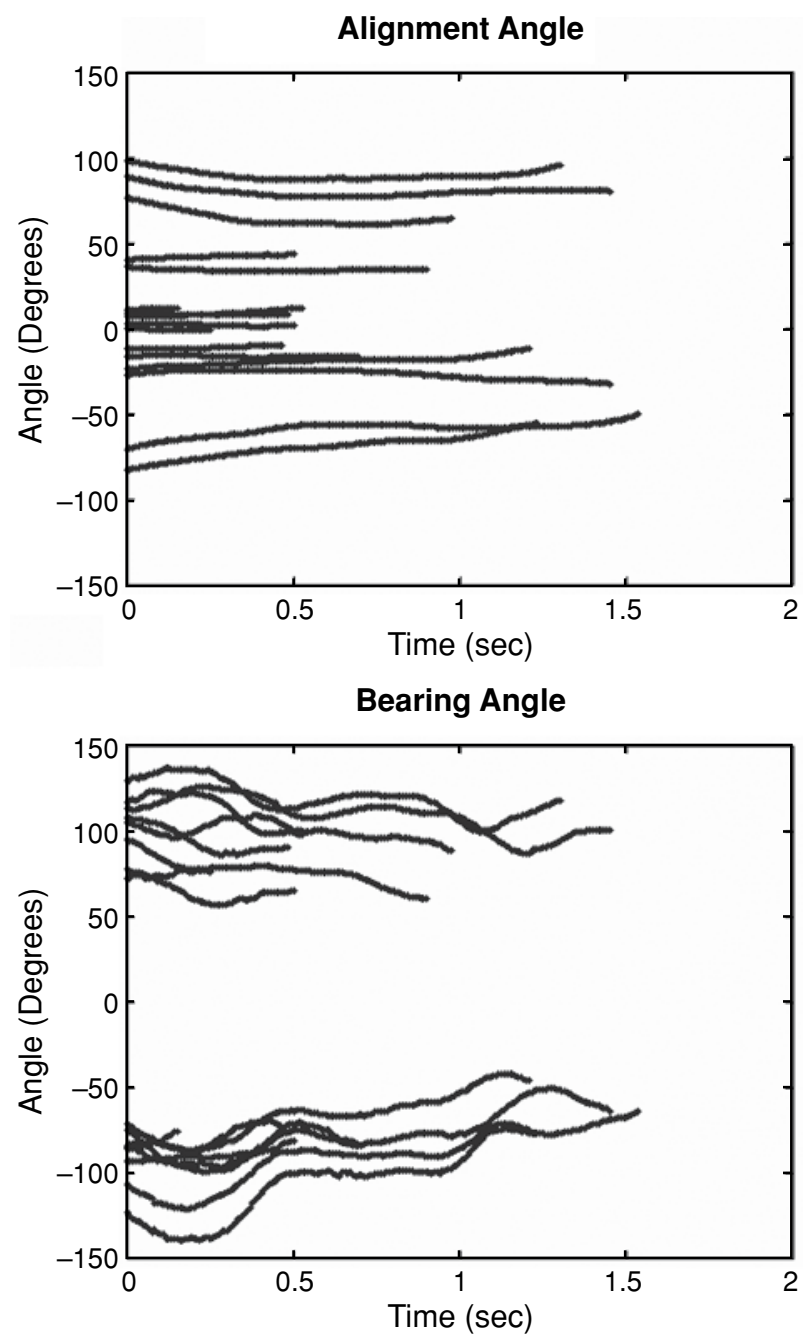

Figure 4. Comparison of two possible lateral control variables with a subset of 16 typical trials. (A) Alignment angle (gaze angle projected in the $X Z$ ground plane) remains relatively constant. (B) Bearing angle (direction of pursuit relative to target alignment) varies significantly more than does alignment angle.

position (Figure 6B shows 16 typical trials). Variance was determined by fitting regression lines to $\cot \alpha$ versus time functions. In other words, the distance between the fielder and the ball position along a virtual horizontal image plane (as shown in Figure 1B) consistently decreases at a constant rate. This projection plane is analogous to a camera with a fixed focal length that is mounted at the fielder's head with its image plane parallel to the ground, moving up and down as head height changes. This is geometrically equivalent to the interception of fly balls, in which the vertical image plane moves forward and backward as the fielder moves in depth. The position of the ball in this image plane is determined by the direction of the gaze vector from head to ball. We also found no notable individual differences among fielders, with both the expert and the less skilled fielders maintaining robust optical speed constancy. The OAC control model of maintaining 

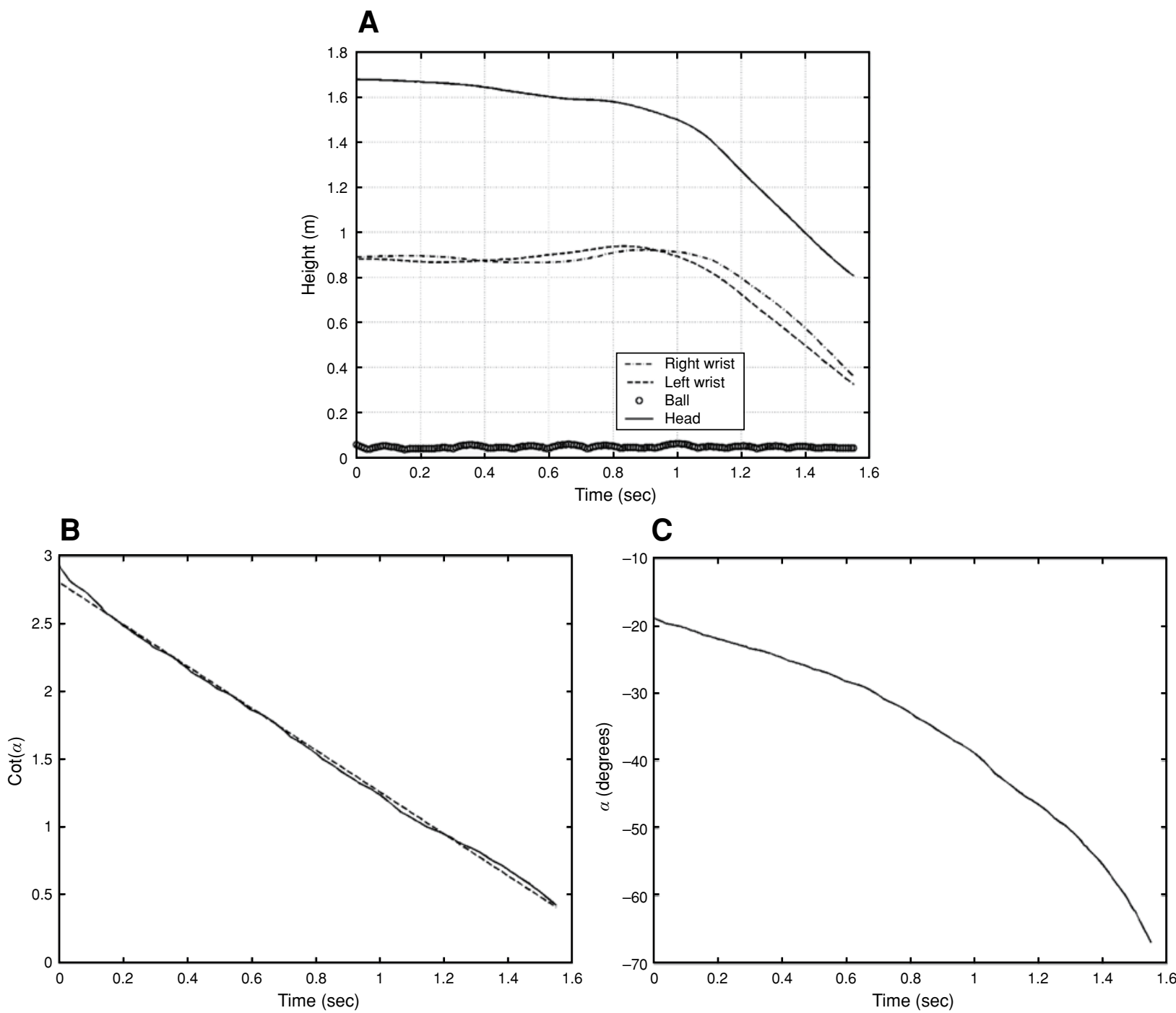

Figure 5. Head height, cotangent of the declination angle (cot $\alpha$ ), and actual declination angle ( $\alpha$ ) on a typical trial. (A) Ongoing heights of the ball and a fielder's head and wrists on a typical trial. (B) Even though the fielder's head height varies considerably, cot $a$ decreases linearly with time. The dashed line indicates the best-fitting regression line. (C) $a$ follows a cotangent function, which does not vary linearly with time during the trial.

a constant rate of change of $\cot \alpha$ accounted for medians of $99.1 \%, 97.9 \%$, and $97.9 \%$ variance in optical ball position for the three fielders, respectively.

The OAC mechanism does not require that processing of optical speed take place in a vertical or horizontal image plane, but the constantly changing tangent and cotangent functions are represented linearly in respective vertical and horizontal image planes. If processing of ball speed takes place in a plane perpendicular to gaze direction, then simple tangent and cotangent transformations can simplify control of optical ball speed.

\section{Optical Linearity Analysis (LOT Strategy for Interception of Ground Balls)}

Optical linearity is analyzed using two essentially equivalent metrics with identical results. All calculations are performed in the image plane perpendicular to gaze direction. In both metrics, gaze direction to the ball is compared to previous gaze direction $(0.0083 \mathrm{sec}$ earlier). Change in vertical optical angle $(\Delta \alpha)$ is recorded as well as lateral change in distance. A constant focal distance is assumed, and lateral change along the image plane is converted into lateral angular change $(\Delta \beta)$. Next, the small changes $\Delta \alpha$ and $\Delta \beta$ are integrated to determine vertical gaze angle $\alpha$ and lateral angle $\beta$. In Figure 7A, $\alpha$ and $\beta$ are plotted over time and are very linear. An increase in $\beta$ is due principally to the fielder's running laterally. A decrease in $\alpha$ is due to both head tilting and the fielder's running in depth toward the interception point.

In the top and right axes, Figure 7A shows the trajectory of ball image relative to background in an image plane that rotates to remain perpendicular with fielder gaze direc- 

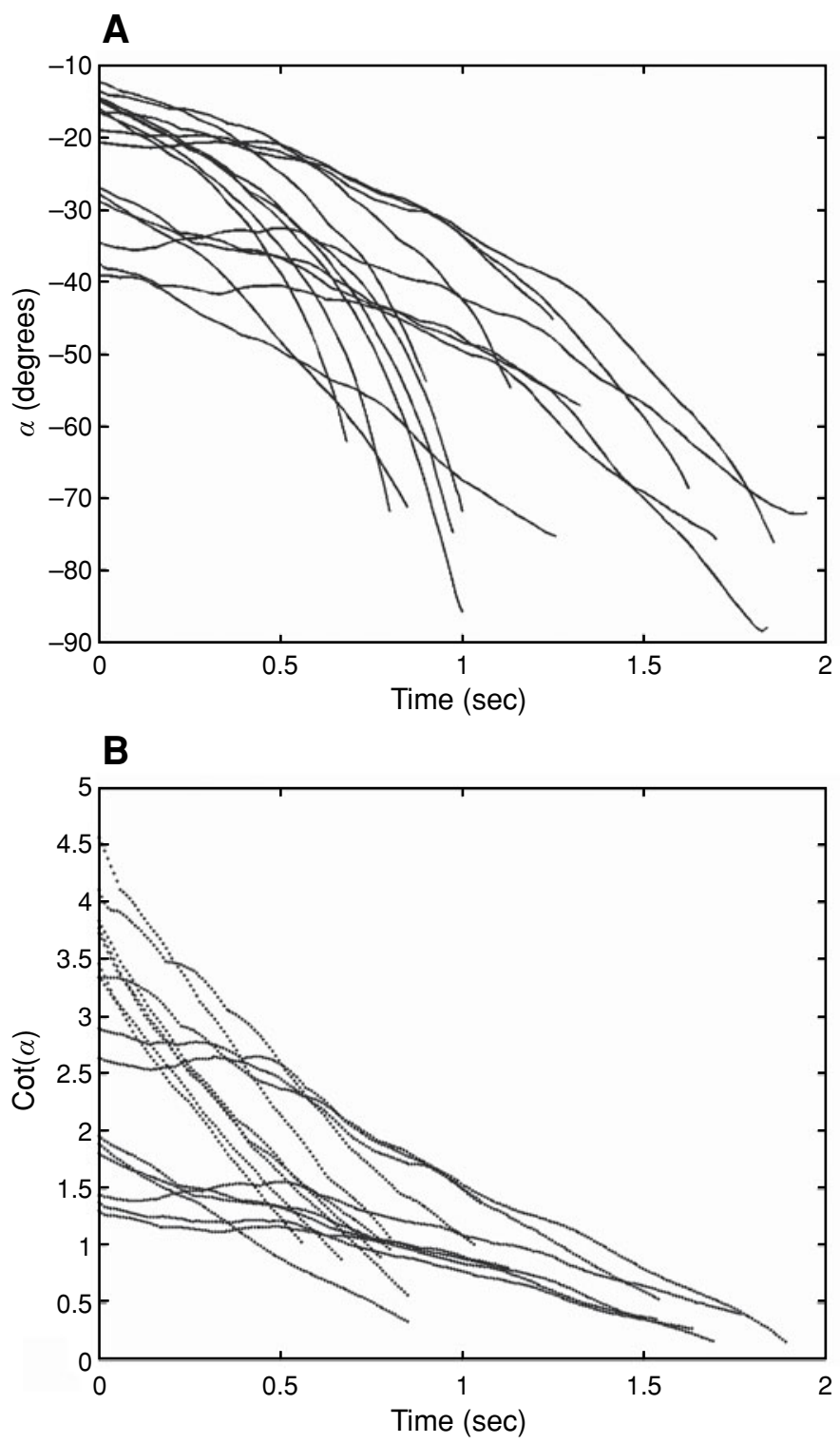

Figure 6. Results from 16 typical trials. (A) Declination angle ( $\alpha$ ) decreases during a trial but does not follow a smooth, straight-line function. (B) Cotangent of the declination angle $(\cot \alpha)$ follows a smooth, straight-line function.

tion. This projection plane remains perpendicular to the body-centered $z$-axis shown in Figure 2. The right $y$-axis of the figure represents cumulative vertical optical projection distance over which the ball image moves relative to background. The top $x$-axis represents cumulative lateral optical distance over which the ball image moves relative to background. When the interception task begins, optical ball position is set to $(0,0)$, so all trajectories start from the origin. Because $\Delta \alpha$ and $\Delta \beta$ are very small, tangents of these angles are nearly identical to the original angles. Thus, optical path in the projection plane using a constant focal distance is identical to the path using optical angles, within resolution of the graph.
The linear relationship between lateral and vertical ball image movements along the viewer-based image plane accounted for a median of $98.2 \%$ of variance on all trials, regardless of whether optical angle data or projection plane data were used in the statistical analysis. On every trial, the fielders navigated to maintain a linear optical trajectory in a rotating viewer-based image plane.

Figure 7B illustrates how the optical size of the ball increases as the fielder approaches the interception point. Here, the best-fitting linear approximation always remains within a ball width of observed optical trajectory. The increased size of the ball image allows its center to deviate more from the linear ideal while its edge remains close, 

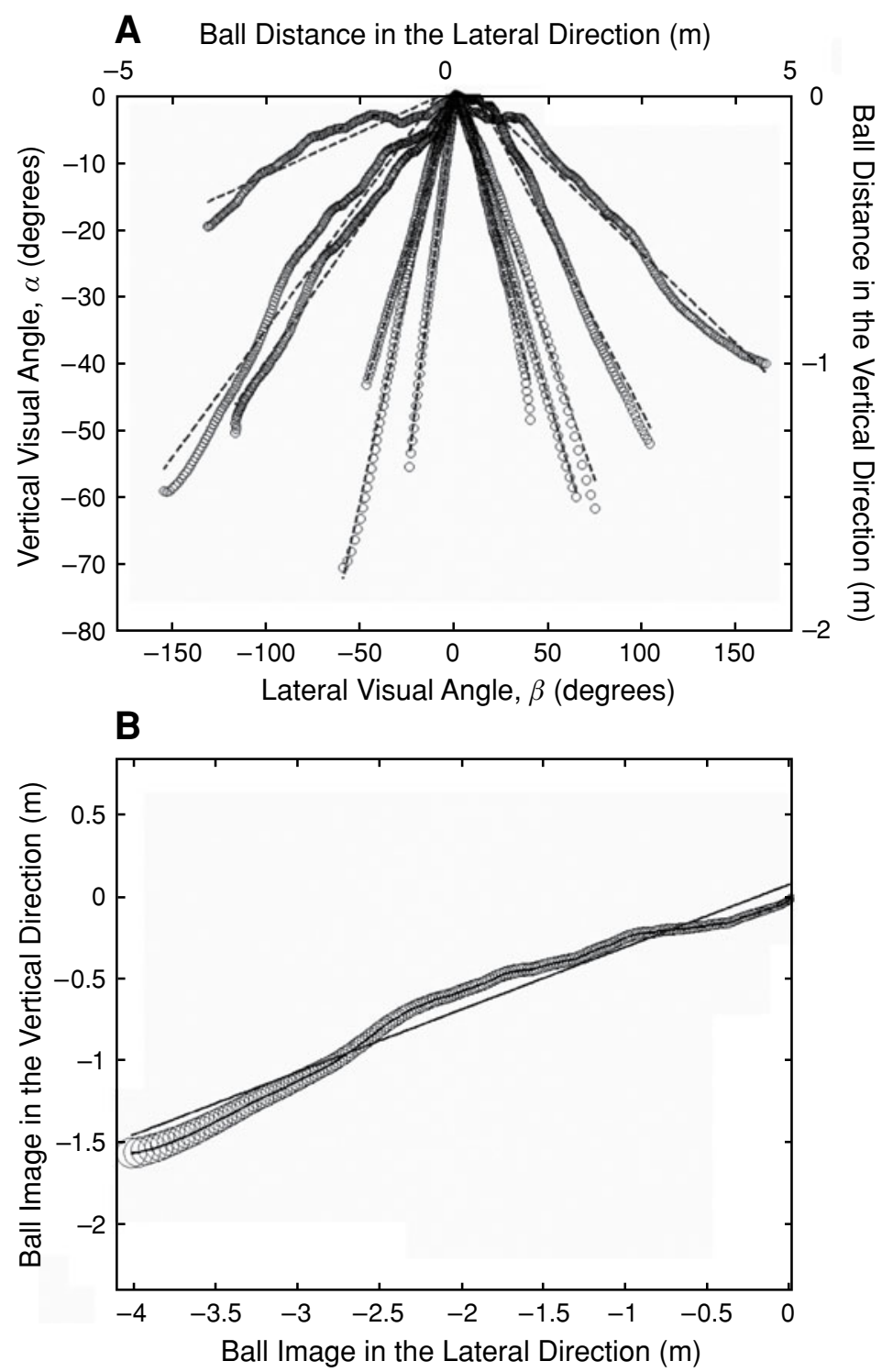

Figure 7. Typical optical trajectories in the rotated viewer-based image plane for intercepted ground balls. Change in ball position along the image plane remains quite linear on all trials. (A) Vertical and lateral optical ball position angles ( $\alpha$ and $\beta$, respectively) in the viewer-based image plane. The bottom and left axes indicate $\beta$ and $\alpha$, respectively, defined as the sum of all instantaneous changes in optical ball position relative to the background. The top and right axes, respectively, indicate the lateral and vertical positions of the optical projection of the moving ball relative to its initial position on an image plane that rotates to be perpendicular with fielder gaze. Focal length was chosen at $1.5 \mathrm{~m}$, the approximate eye height of a moving fielder. (B) Plot of a typical trajectory showing how the optical size of the ball increases as the fielder approaches the interception point. The best-fitting linear solution remains within a ball width of the edge of the ball.

which could explain deviations in linearity near the end of trajectories.

\section{CONCLUSION}

In this work, we confirm that fielder behavior is consistent with use of the same perceptual control heuristics for intercepting both moving airborne targets and ground-based ones. Despite vast differences in the physical behavior of such targets, in both cases fielders maintain constancy of optical speed (via OAC) and angle of the target (via LOT). The fundamental difference is that, in the case of airborne targets, fielders move to cancel out changes in the tangent of vertical optical angle - the algebraic equivalent to keep- 
ing the target image moving at a constant speed along a virtual vertical image plane. In the case of ground-based targets, fielders move to cancel out changes in the cotangent of vertical optical angle - the algebraic equivalent to keeping the target image moving at a constant speed along a virtual horizontal image plane. The OAC mechanism does not require that processing of optical speed take place in such vertical or horizontal image planes, but simply that the temporal pattern follow the equivalent constant change in tangent and cotangent functions.

The data also support a parallel strategy to keep the ball image moving along a linear optical path. In intercepting both airborne and ground balls, a fielder moves laterally along a path that maintains approximate alignment with the ball, resulting in a linear optical trajectory along a viewer-based image plane that rotates to remain perpendicular with the gaze of the fielder. The fielder could accomplish this by keeping the ball image centered on a viewer-based reference frame while moving to produce local background flow in a constant direction.

This work supports the simultaneous use of multiple heuristics during navigation to intercept moving targets. This is consistent with research showing that observers use multiple sources of information to determine relative location of objects in static viewing conditions (Landy, Maloney, Johnston, \& Young, 1995). The use of multiple heuristics is also consistent with previous findings that both optical heuristics break down when targets become uncatchable (Shaffer \& McBeath, 2002).

Past research showed that observers can use angle of declination to assess distance to objects and make judgments about locations in space (Ooi et al., 2001). The present study extends those findings in showing that observers can also dynamically control changes in angle of declination to guide them during ground-based interception tasks.

Past research in interceptive behavior has been limited by the difficulty of exactly measuring ongoing angles between observers and targets. The present use of a rapidrefresh, multicamera, 3-D motion capture system provided indisputable high-resolution measurements. With this procedure, we were able to confirm behavior consistent with the use of the optical control heuristics with the highest rate of variance accounted for to date.

Finally, the work has applied relevance. For example, in robotics our findings are directly applicable to navigation control algorithms being used to develop autonomous mobile robots. The simple geometric principles are easily programmed using optical control variables and can guide mobile robotic aids to navigate naturally toward or away from moving objects, as is needed in collision avoidance for the visually impaired. In short, the findings confirming the strategy of maintaining optical speed and direction for both airborne and ground-based targets support the proposal that these mechanisms are universal and domain independent.

\section{REFERENCES}

Aboufadel, E. (1996). A mathematician catches a baseball. American Mathematics Monitor, 103, 870-878.
BABler, T. G., \& DANNEMILler, J. L. (1993). Role of image acceleration in judging landing location of free-falling projectiles. Journal of Experimental Psychology: Human Perception \& Performance, 19, 15-31.

Beall, A. C., \& Loomis, J. M. (1997). Optic flow and visual analysis of the base-to-final turn. International Journal of Aviation Psychology, 7, 201-223.

BrancazIO, P. (1985). Looking into Chapman's homer: The physics of judging a fly ball. American Journal of Physics, 53, 849-855.

Chapman, S. (1968). Catching a baseball. American Journal of Physics, 36, 868-870.

Cutting, J. E., \& WANG, R. F. (2000). Heading judgments in minimal environments: The value of a heuristic when invariants are rare. Perception \& Psychophysics, 62, 1146-1159.

FAJEN, B. R., \& WARREN, W. H. (2004). Visual guidance of intercepting a moving target on foot. Perception, 33, 689-715.

Landy, M. S., Maloney, L. T., Johnston, E. B., \& Young, M. (1995). Measurement and modeling of depth cue combination: In defense of weak fusion. Vision Research, 35, 389-412.

LEE, D. N. (1998). Guiding movement by coupling taus. Ecological Psychology, 10, 221-250.

LeE, D. N., \& RedDISH, P. E. (1981). Plummeting gannets: A paradigm of ecological optics. Nature, 293, 293-294.

Lenoir, M., Musch, E., Janssens, M., Thiery, E., \& Uyttenhove, J. (1999). Intercepting moving objects during self-motion. Journal of Motor Behavior, 31, 66-67.

MARKen, R. S. (1997). The dancer and the dance: Methods in the study of living control systems. Psychological Methods, 2, 436-446.

Marken, R. S. (2001). Controlled variables: Psychology as the center fielder views it. American Journal of Psychology, 114, 259-281.

MARKEN, R. S. (2005). Optical trajectories and the informational basis of fly ball catching. Journal of Experimental Psychology: Human Perception \& Performance, 31, 630-634.

McBeath, M. K., Shaffer, D. M., \& Kaiser, M. K. (1995). How baseball outfielders determine where to run to catch fly balls. Science, 268, 569-573.

McBeath, M. K., Shaffer, D. M., \& Kaiser, M. K. (1996). On catching fly balls. Science, 273, 256-259.

McBeath, M. K., Shaffer, D. M., Morgan, S. E., \& Sugar, T. G. (2002). Lack of conscious awareness of how we navigate to catch baseballs. Toward a Science of Consciousness, 5, 84.

McBeath, M. K., Shaffer, D. M., \& Sugar, T. G. (2002). Catching baseball pop flies: Individual differences in aggressiveness and handedness. Abstracts of the Psychonomic Society, 7, 103.

McBeath, M. K., Sugar, T. G., Thompson, M. J., \& Mundhra, K. (2003). Catching ground balls: Optical control heuristics used by humans and robots support a unified fielder theory. Journal of Vision, 3, 543.

McLeod, P., \& Dienes, Z. (1993). Running to catch the ball. Nature, 362, 23.

McLeod, P., \& Dienes, Z. (1996). Do fielders know where to go to catch the ball or only how to get there? Journal of Experimental Psychology: Human Perception \& Performance, 22, 531-543.

McLeod, P., ReEd, N., \& Dienes, Z. (2003). How fielders arrive in time to catch the ball. Nature, 426, 244.

Milner, A., \& Goodale, M. (1995). The visual brain in action. Oxford: Oxford University Press.

Mundhra, K., Sugar, T. G., \& McBeath, M. K. (2003, September). Perceptual navigation strategy: A unified approach to interception of ground balls and fly balls. Paper presented at the IEEE International Conference on Robotics and Automation, Taipei.

Mundhra, K., Suluh, A., Sugar, T. G., \& McBeath, M. K. (2002). Intercepting a falling object: Digital video robot. In Proceedings of the 2002 IEEE International Conference on Robotics and Automation (pp. 2060-2065). Washington, DC.

OoI, T. L., Wu, B., \& HE, Z. J. (2001). Distance determined by the angular declination below the horizon. Nature, 414, 197-200.

Oudejans, R. R. D., Michaels, C. F., \& Bakker, F. C. (1997). The effects of baseball experience on movement initiation in catching fly balls. Journal of Sports Sciences, 15, 587-595.

Pollack, H. N. (1995). Play ball! Science, 268, 1681.

REGAN, D. (1997). Visual factors in hitting and catching. Journal of Sports Sciences, 15, 533-558. 
Regan, D., \& Gray, R. (2000). Visually guided collision avoidance and collision achievement. Trends in Cognitive Sciences, 4, 99-107.

SaXberG, B. V. H. (1987). Projected free fall trajectories: II. Human experiments. Biological Cybernetics, 56, 177-184.

Shaffer, D. M., Krauchunas, S. M., EdDy, M., \& McBeath, M. K. $(2004)$. How dogs navigate to catch Frisbees. Psychological Science, $15,437-441$

ShafFer, D. M., \& McBeath, M. K. (2002). Baseball outfielders maintain a linear optical trajectory when tracking uncatchable fly balls. Journal of Experimental Psychology: Human Perception \& Performance, 28, 335-348.

Shaffer, D. M., \& McBeath, M. K. (2005). Naive beliefs in baseball: Systematic distortion in perceived time of apex for fly balls. Journal of Experimental Psychology: Learning, Memory, \& Cognition, 31, 1492-1501.

Sugar, T. G., \& McBeath, M. K. (2001a). Robotic modeling of mobile ball-catching as a tool for understanding biological interceptive behavior. Behavioral \& Brain Sciences, 24, 1078-1080.

Sugar, T. G., \& McBeath, M. K. (2001b, June). Spatial navigation algorithms: Applications to mobile robotics. Paper presented at the Proceedings of the 6th Vision Interface Annual Conference, Ottawa.
Sugar, T. G., McBeath, M. K., Suluh, A., \& Mundhra, K. (2006). Mobile robot interception using human navigational principles: Comparison of active versus passive tracking algorithms. Autonomous Robots, 21, 43-54.

Suluh, A., Mundhra, K., Sugar, T. G., \& McBeath, M. K. (2002). Spatial interception for mobile robots. In Proceedings of the 2002 IEEE International Conference on Robotics and Automation (pp. 4263-4268). Washington, DC.

VICON (2003). Motion capture system. Available at www.vicon.com.

Warren, W. H., Kay, B. A., Zosh, W. D., Duchon, A. P., \& Sahuc, S. (2001). Optic flow is used to control human walking. Nature Neuroscience, 4, 213-216.

WATTS, R. G., \& BAHILl, A. T. (2000). Keep your eye on the ball: Curve balls, knuckleballs, and fallacies of baseball (2nd ed.). New York: Freeman.

(Manuscript received August 22, 2004; revision accepted for publication March 18, 2006.) 